\title{
The Mycology Collections Portal (MyCoPortal)
}

\author{
Andrew N. Miller ${ }^{1}$ and Scott T. Bates ${ }^{2}$
}

${ }^{1}$ Illinois Natural History Survey, University of Illinois, Champaign, IL, 61820, USA; corresponding author e-mail: amiller7@illinois.edu

${ }^{2}$ Department of Biological Sciences, Purdue University Northwest, Westville, IN 46391, USA

Abstract: The Mycology Collections Portal (MyCoPortal) was established in 2011 to offer a simple, economic solution for institutions worldwide to maintain and serve their fungal collection data online. Although the MyCoPortal functions primarily as a web portal for collections to upload their specimen metadata and images, it also provides data packaging (e.g. checklists), visualization (mapping and timelines), educational material (identification keys, quizzes) and easy access to DwC formatted data sets as downloadable csv files. The MyCoPortal currently serves over 3.6M records from 84 institutions worldwide.

Key words: data portal, database, fungaria, fungi, genetic data, georeferencing, natural history collections, specimens.

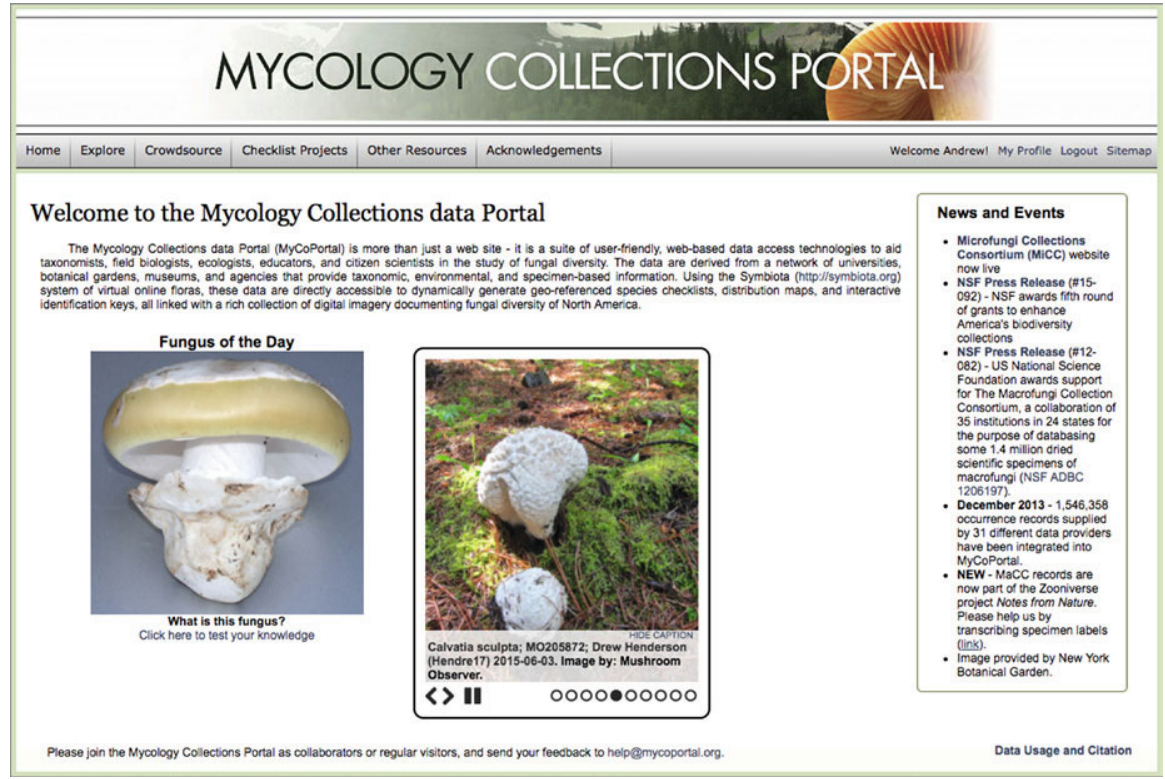

Fig. 1. The Mycology Collections Portal home page.

The Mycology Collections Portal (MyCoPortal, http://mycoportal.org; Fig. 1) is part of a network of web-based biodiversity resources built on the Symbiota data management platform (http://www. symbiota.org), which uses open source, modular software to run an underlying SQL database on a webserver (Gries et al. 2014). Symbiota allows for the creation of collaborative, specimen-based virtual biota web portals that include features such as species pages, taxonomic checklists, identification keys, image libraries, and distribution maps. These portals also interact with outside sources, for example through linkages to external databases (e.g. genetic repositories) or by serving as data aggregators (e.g. integration of crowd-sourced citizen scientist observations). Symbiota portals now serve biodiversity data online from over 750
US from 2012-2015 and resulted in the addition of $1.2 \mathrm{M}$ macrofungal records. $\mathrm{MaCC}$ was followed by the Microfungi Collections Consortium (MiCC - http:// www.microfungi.org), another NSF ADBC grant for 2.8 M US\$ awarded to Andrew Miller (ILLS), who is presently directing the digitization efforts of 38 institutions from 2015-2019 that has resulted in the digitization of an additional $2.1 \mathrm{M}$ microfungal records. The MyCoPortal currently serves over 3.3 M records of voucher specimens, along with an additional 280 000 observational records, from 84 institutions throughout the World, but predominately from North America (http://www.microfungi.org/index.php/ mycoportal). These data include over 118000 type specimens and over 300 different exsiccate sets. With the completion of the MaCC and MiCC projects, nearly all North American fungal specimens housed in US fungaria will be digitized and available through the MyCoPortal.

The MyCoPortal's mission is to create an immense networked resource to aid mycological research and education by distributing online information generated from core users (e.g. institutional collections) to researchers and educators throughout the World. The primary goal is to provide open source, web-based tools for data curation and collections management that facilitates documentation (through both vouchered specimens and observations) of fungal species occurrences, distributions, and phenology. Specimen records can be linked to static checklists (e.g. serving as vouchers for local floristic studies), images (e.g. photographs \begin{abstract}
efforts of 35 institutions throughout the
\end{abstract}
40 portals containing $~ 37$ million specimen plants, invertebrates and vertebrates.

The MyCoPortal was created in 2011 to hest digitized data on non-lichenized fungi, initially sourced from the Denver Botanic Gardens (NY) and the Illinois Natural History Survey (ILLS). This early effort enabled successful funding of a 3 M US\$ National Science Foundation (NSF) grant in the Advancing Digitization of Biological Collections (ADBC) program, which was awarded to Barbara Thiers (NY) in 2012. This project, the Macrofungi Collections Consortium ${ }^{1}$, directed the digitization 
Locus: Tomentella sublilacina voucher UC2022964 18S ribosomal RNA gene, partial sequence; internal transcribed spacer 1, 5.8S ribosomal RNA gene, and internal transcribed spacer 2, complete sequence; and 28S ribosomal RNA gene, partial sequence

URL: http: www.ncbi.nIm.nih.gov/nuccore/KP814555.1

Notes:

Fig. 2. A record illustrating genetic data in the MyCoPortal.

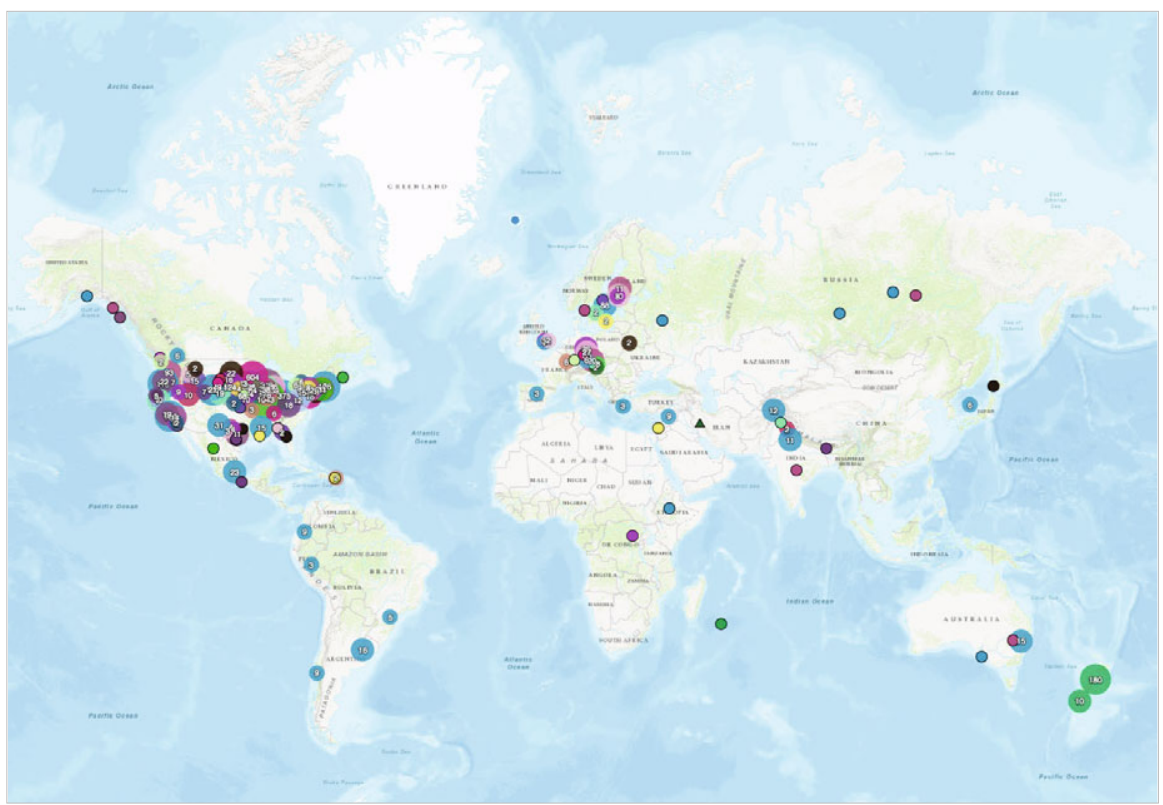

Fig. 3. Spatial map showing the worldwide distribution of Puccinia graminis generated from 5441 specimens from 28 collections in the MyCoPortal.

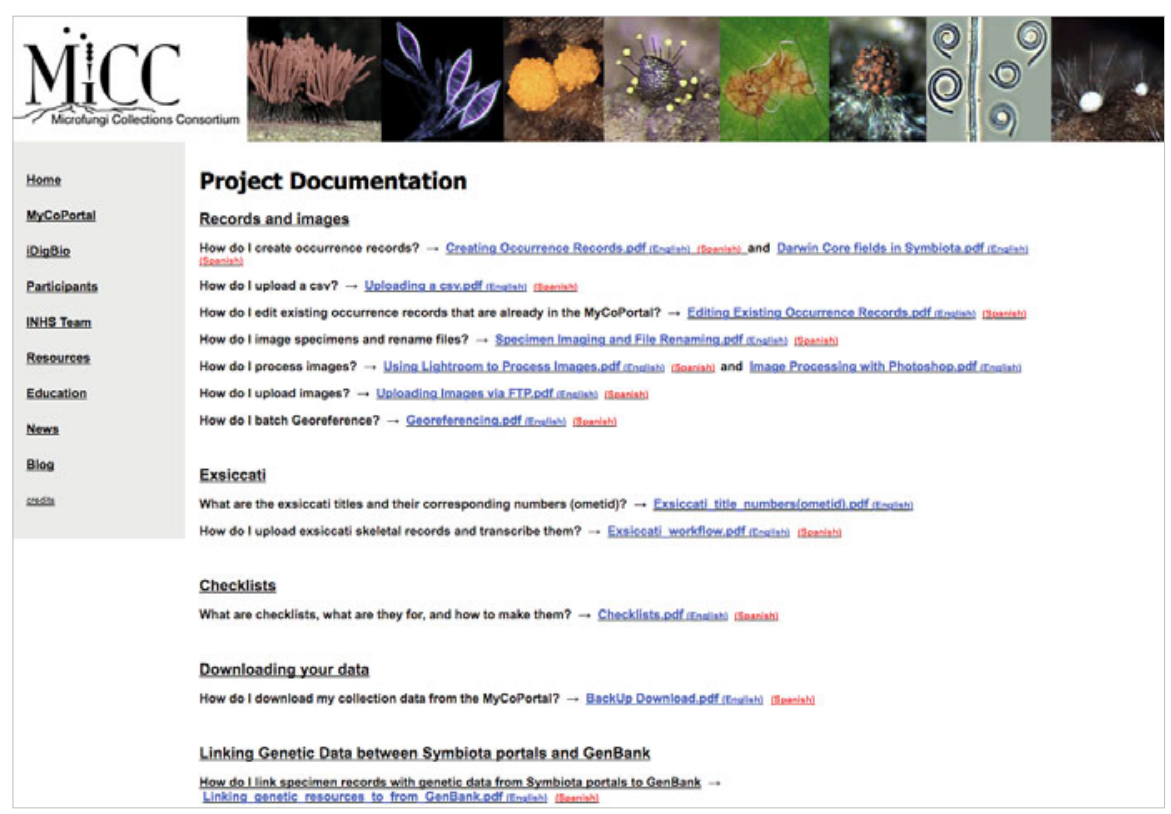

Fig. 4. MyCoPortal project documentation.

from the field, illustrations, and ancillary notes), and DNA sequence data (e.g. GenBank accessions; Fig. 2). MyCoPortal specimens specifically linked to records in GenBank now also serve as metadata for those accessions through the NCBI's LinkOut feature (see e.g. 'LinkOut to external resources' under https:// www.ncbi.nlm.nih.gov/nuccore/kp814555).
The MyCoPortal's organizational structure gives autonomy to individual institutions; these operating as separate, independent collections that can serve (free of cost) and control their own data (e.g. by assigning permissions that only allow editing rights for specific users), but with all collections working together within a general network to build a common, comprehensive database (viz. a 'cloud' platform for fungal specimen data). Collections maintain easy access to their data sets through simple downloadable csv backup files in $\mathrm{DwC}$ format. The ease of assembling and visualizing datasets by end users has made the MyCoPortal extremely useful for curating data, creating specialized mycofloras, as well as discovering "lost" specimens (Delgado \& Koukol 2016). Recent development of MyCoPortal's spatial module now allows for a range of new visualization options to aid in data analysis, including for example: capacity to map species ranges (Fig. 3) and sporophore production patterns incorporating user inputted reference layers (e.g. biotic community data); ability to produce heat maps (e.g. reflecting levels of species diversity throughout the World); and potential to model habitat suitability or possible climate change impacts. Existing modules currently provide users with a number of exciting possibilities for interaction with MyCoPortal data. For example, through the public search engine users can generate dynamic checklists for an area of interest (e.g. a region defined by a bounding box), display a classification according to different taxonomic thesauri (e.g. Index Fungorum or MycoBank), and even connect to an interactive key for the generated list. MyCoPortal is a simple, economic, online resource for fungaria to curate, maintain and serve their fungal collection data. Ample documentation on how to use the MyCoPortal can be found on the Microfungi Collections Consortium webpage (http:// www.microfungi.org; Fig. 4).

\section{ACKNOWLEDGEMENTS}

The development and success of the MyCoPortal would not have been possible without the support of the National Science Foundation program Advancing Digitization of Biological Collections through awards for digitizing macrofungi (DBI-1206197) and microfungi (DBI-15-02735).

\section{REFERENCES}

Delgado G, Koukol O (2016) Microfungi from

Nicaragua in a historical collection kept at the herbarium of the Charles University in Prague. Cryptogamie Mycologie 37:15-36.

Gries C, Gilbert EE, Franz NM (2014) Symbiota - a virtual platform for creating voucherbased biodiversity information communities. Biodiversity Data Journal 2: e1114. https://doi. org/10.3897/BDJ.2.e1114 